AperTO - Archivio Istituzionale Open Access dell'Università di Torino

\title{
Calcium signals: analysis in time and frequency domains
}

\section{This is a pre print version of the following article:}

Original Citation:

\section{Availability:}

This version is available http://hdl.handle.net/2318/133521

since 2021-04-10T11:18:24Z

Published version:

DOI:10.1016/j.jneumeth.2011.05.009

Terms of use:

Open Access

Anyone can freely access the full text of works made available as "Open Access". Works made available under a Creative Commons license can be used according to the terms and conditions of said license. Use of all other works requires consent of the right holder (author or publisher) if not exempted from copyright protection by the applicable law. 


\title{
Calcium signals: analysis in time and frequency domains
}

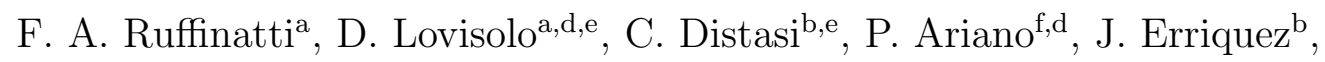 \\ M. Ferraro ${ }^{\mathrm{c}, \mathrm{e}}$ \\ ${ }^{a}$ Università di Torino, Dipartimento di Biologia Animale e dell'Uomo, Torino, Italy \\ ${ }^{b}$ Università del Piemonte Orientale, Dipartimento di Scienze Chimiche, Alimentari, \\ Farmaceutiche e Farmacologiche, Novara, Italy \\ ${ }^{c}$ Università di Torino, Dipartimento di Fisica Sperimentale, Via P. Giuria 1, 10125 \\ Torino, Italy \\ ${ }^{d}$ NIS, Centre of Excellence for Nanostructured Interfaces and Surfaces, University of \\ Turin, Italy \\ ${ }^{e}$ NIT, Neuroscience Institute of Turin, Italy

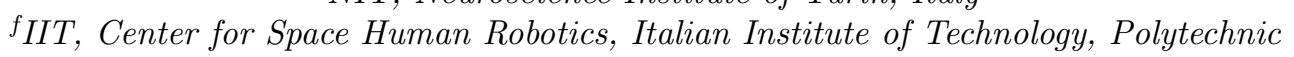 \\ University of Turin, Italy
}

\begin{abstract}
Cytosolic calcium signals play important roles in processes such as cell growth and motility, synaptic communication and formation of neural circuitry. These signals have complex time courses and their quantitative analysis is not easily accomplished; in particular it may be difficult to evidence subtle differences in their temporal patterns. In this paper, we use wavelet analysis to extract information on the structure of $\left[\mathrm{Ca}^{2+}\right]_{c}$ oscillations. To this aim we have derived a set of indices by which different $\left[\mathrm{Ca}^{2+}\right]_{c}$ oscillatory patterns and their change in time can be extracted and quantitatively evaluated. This approach has been validated with examples of experimental recordings showing changes in oscillatory behavior in cells stimulated with a calcium-releasing agonist.
\end{abstract}

Keywords:

computational methods, wavelet analysis, calcium signals

Email addresses: federicoalessandro.ruffinatti@unito.it (F. A. Ruffinatti), davide.lovisolo@unito.it (D. Lovisolo), carla.distasi@pharm.unipmn. it (C. Distasi), paolo.ariano@gmail.com (P. Ariano), jessica.erriquez@pharm.unipmn.it (J. Erriquez), ferraro@ph.unito.it (M. Ferraro) 


\section{Introduction}

A large array of cellular functions is under the control of fine and complex changes in the free cytosolic calcium concentration, $\left[\mathrm{Ca}^{2+}\right]_{c}$. These changes convey information on the specific status of the metabolic machinery and on the signals impinging on the cell itself, and this information can be coded both in amplitude and frequency (Oike et al., 1994; Prank et al., 2000). Additionally, these intracellular events are usually compartmentalized, i.e. they are restricted to specific subcellular domains, with different compartments showing different patterns of $\mathrm{Ca}^{2+}$ signalling (Frey et al., 2000; Goldberg and Yuste, 2005; Raymond and Redman, 2006). In nerve cells, the role of these intracellular signals is of particular relevance: they can regulate a wide set of processes, from cell to cell communication to integration of information at the cell body and to transcriptional events. Moreover, they have specific roles at defined developmental stages, such as in the growth, orientation and stabilization of neuronal processes (dendrites and axons) and in the correct formation of neuronal circuitry (Wen and Zheng, 2006).

The generation of these cytosolic signals, usually showing an oscillatory pattern, is the result of a convergent and tightly regulated set of activations and deactivations of calcium import and export mechanisms, together with pathways involving exchange of the ion between the cytosol and intracellular compartments (Uhlén and Fritz, 2010). The resulting responses are often of complex time course, and their quantitative analysis is not so obvious. In many cases, they appear to be non-periodic and it may be difficult to evidence subtle differences in signal patterns by qualitative or semiquantitative observation. On the other hand, evidencing statistical differences may be necessary when analyzing changes in activity following treatments with different agonists (neurotransmitters, hormones, growth factors, guidance molecules) or comparing activity in different subcellular domains (soma, growth cone, etc.). Some simple and in some cases effective approaches have been developed (Constantin et al., 2009), but, in general, spectral analysis is mandatory. Fourier transform has been widely used (see e.g. Uhlén, 2004); however, it provides a mean of analysis solely in the space of frequencies. To overcome this limitation, some authors have employed wavelet analysis (Gorbunova and Spitzer, 2002; Suzuki et al., 2002; Wegner et al., 2006), that takes into account the local frequency composition of the signal and enables the signal to be analyzed both in frequency and time spaces. On the other hand, many applications of wavelet analysis do not provide quantitative parame- 
ters to describe changes in the frequency composition of oscillatory patterns following stimulation protocols. This is a relevant issue, since such changes may provide relevant information on the kinetics of the mechanisms involved.

In this paper, we introduce a more sophisticated approach to wavelet analysis, and show that it can be of significant help in extracting information from traces in which changes in the pattern of $\mathrm{Ca}^{2+}$ oscillations cannot be evidenced by qualitative observation. To this purpose, starting from the standard wavelet analysis, we have derived a set of indices by which different $\left[\mathrm{Ca}^{2+}\right]_{c}$ oscillatory patterns and their change in time can be extract and quantitatively evaluated. The potential usefulness of this approach can be extended to other contexts, such as the analysis of differences between activities in subcellular domains of the same neuron, and, more generally, of the same cell.

\section{Mathematical preliminaries}

A standard method to analyze a signal $f(t)$ is via its Fourier transform which, however, provides information only on the frequencies making up the signal: that is to say that although, in principle, it is possible to determine all the frequencies present in a signal, the time at which they occur cannot be determined. To overcome this problem in the past decades several solutions have been developed to represent a signal in the time and frequency domain at the same time.

A typical example is the windowed Fourier transform where the kernel of the Fourier transform is multiplied by a temporal window, say $g_{a}(t-b)$, where the parameter $a$ measures the width of the window, and the parameter $b$ is used to translate the window over the whole time domain. The resulting transform is called the Gabor transform (windowed Fourier transform) (Lokenath, 1998).

The width of the window, determined by $a$, provides a trade-off between frequency and time resolution: large windows (i.e. large $a$ ) give high resolution of frequency and low time resolution, whereas narrow windows improve time resolution but provide a less accurate frequency representation. In other words a small $a$ gives accurate information on the time course of the signal but it may lead to a coarse frequency representation, possibly losing relevant information on the structure of the signal itself; conversely, a large $a$ provides an accurate representation of the signal structure but important events in the time course of the signal may be overlooked. 
The problem of achieving good time resolution for the high frequency transients and good frequency resolution for low frequency components can be solved with the use of wavelets, a family of functions constructed from translations and dilations of a single function called the "mother wavelet" $\psi$ (for a clear historical introduction see Lokenath (1998) and, for an in depth treatment, Daubechies (1992) and Mallat (1999): the literature on wavelet is virtually unlimited). This function must satisfy certain technical conditions (see, for instance, Antonini et al., 1992), among which $\int \psi(t) d t=0$, so that $\psi$ must exhibit some oscillations and a rapidly decreasing trend. The set of wavelets is obtained by the formula

$$
\psi^{(a, b)}(t)=\frac{1}{|a|^{1 / 2}} \psi\left(\frac{t-b}{a}\right),
$$

where $a$ is a scaling parameter which measures the degree of compression or scale, and $b$ a translation parameter which determines the time location of the wavelet (Daubechies, 1992). If $|a|<1$ the wavelet $\psi^{(a, b)}$ corresponds mainly to higher frequencies, when $|a|>1$ it has a larger time-width than $\psi$ and corresponds to lower frequencies. In other words on a large scale, the resolution is coarse in the time domain and fine in the frequency domain and, as the scale parameter $a$ decreases, the resolution in the time domain becomes finer, while that in the frequency domain becomes coarser. Thus, wavelets have time-widths adapted to their frequencies and this is the main reason for their success in time-frequency analysis.

Functions $\psi^{(a, b)}$ form the kernel of the wavelet transform:

$$
W^{(a, b)}=\frac{1}{|a|^{1 / 2}} \int_{-\infty}^{+\infty} \psi^{*}\left(\frac{t-b}{a}\right) f(t) d t,
$$

where $*$ denotes complex conjugation.

Here we have chosen the Morlet function as mother wavelet (Goupillaud et al., 1984):

$$
\psi(t)=\frac{c_{s}}{\pi^{1 / 4}} \exp \left(-\frac{1}{2} t^{2}\right)\left[\exp (i s t)-k_{s}\right],
$$

where $k_{s}=\exp \left(-\frac{1}{2} s^{2}\right)$ and $c_{s}=\left[1+\exp \left(-s^{2}\right)-2 \exp \left(-\frac{3}{4} s^{2}\right)\right]^{-1 / 2}$ is the normalization constant. Usually the parameter $s$ is taken to be equal or larger than 5, so that $k_{s} \simeq 0$ and $c_{s} \simeq 1$ : (3) becomes

$$
\psi(t) \simeq \frac{1}{\pi^{1 / 4}} \exp \left(-\frac{1}{2} t^{2}\right) \exp (i s t)
$$


and the corresponding graph is shown in Fig. 1.

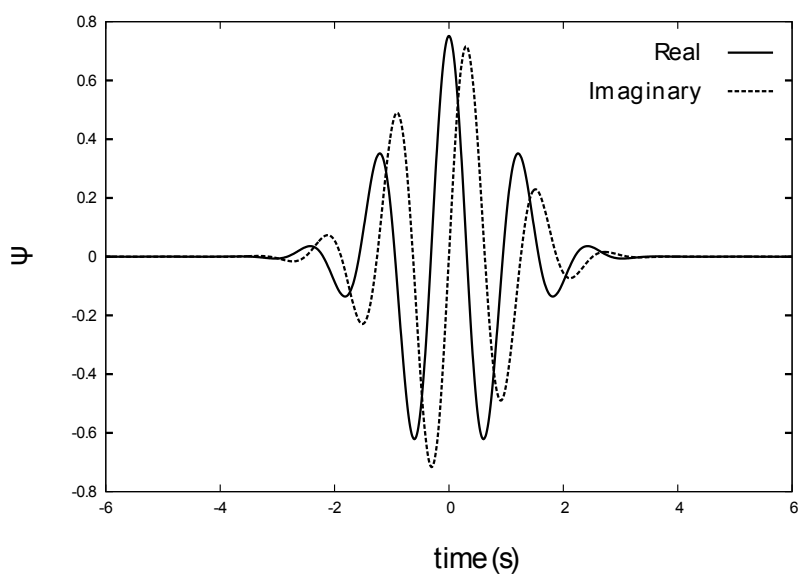

Figure 1: Real and imaginary parts of the Morlet mother wavelet, as given by Eq. (4).

The wavelets transform is

$$
\begin{aligned}
W^{(a, b)} & =\frac{1}{|a|^{1 / 2}} \int_{-\infty}^{+\infty} \psi^{*}\left(\frac{t-b}{a}\right) f(t) d t \\
& =\frac{1}{\left(\pi a^{2}\right)^{1 / 4}} \int_{-\infty}^{+\infty} \exp \left[-\frac{1}{2}\left(\frac{t-b}{a}\right)^{2}\right] \exp \left[-i \frac{s}{a}(t-b)\right] f(t) d t,
\end{aligned}
$$

where we have used $s=5$. It should be noted that $b$ is a time variable and that $s / a$ is related to the distance $T_{p}$ between successive peaks of the mother wavelet (see Fig. 1) by the relation $s / a=2 \pi / T_{p}$ so that it is possible to define a frequency of the wavelet by the relation $\nu=s / 2 \pi a$ (Goupillaud et al., 1984; Mallat, 1999). Thus, in the following the wavelet transform will be denoted by $W(t, \nu)$ : the amplitude (modulus) of $W$ defines the scalogram, a graphical representation of the signal in the time-frequency domain.

\section{Analytical methods}

In this section a method of analysis will be described using recordings of changes in the intracellular free calcium concentration from cultured chick ciliary ganglion (CG) glial cells. The signals represent responses to application to the extracellular solution of the agonist nicotinic acid adenine dinucleotide 
phosphate (NAADP), a molecule that activates calcium release from intracellular stores (Genazzani and Billington, 2002) and that can exert its action when extracellularly applied (Billington et al., 2006). Changes in $\left[\mathrm{Ca}^{2+}\right]_{c}$ have been recorded by means of the fluorescent calcium indicator Fura-2. More details on the experimental procedures will be given in Section 3.4.

A graphical representation of the wavelet transform of one of such signals $f(t)$ is presented in Fig. 2. The signal itself is in the upper box of the figure, and the modulus (amplitude) $|W(t, \nu)|$ of its wavelet transform is displayed in the lower box using an appropriate pseudocolor look-up table. It is known that wavelet transforms in finite time intervals give rise to the so called cone of influence at the edges of the time span of the recording (Torrence and Compo, 1998); here a detrending procedure was used that, ensuring a matching between the start and the end of the signal, can reduce the cone of influence artifact, by removing edge discontinuity; however this procedure does nothing about the most important source of this effect, namely the lack of information on the events occurring before the start and after the end of the recording.

This trace was selected since even from a qualitative observation it can be concluded that it shows a sharp response to the agonist, in the form of a transient oscillatory burst. From the figure it is apparent that $|W(t, \nu)|$ takes its maximum values during the oscillatory burst of $f(t)$, and that outside the areas of these peaks its values are relatively small, except for a low frequency component that corresponds to an overall oscillation of $f(t)$. Furthermore, it can be noted that peaks are more spread out at low frequencies, as expected from wavelet theory: at low frequency the time resolution tends to be less precise (Mallat, 1999). Thus the use of wavelet analysis implies some uncertainty in time (i.e. the exact time at which a specific component can be localized) and this holds mainly for low frequency components. However, the extent of the oscillatory burst is in good agreement with the temporal position of the peaks in the scalogram, thus showing that wavelets provide temporal information about the start and duration of the oscillatory part of the signal.

While $|W(t, \nu)|$ provides information on both the oscillatory structure of the signal and its temporal trend, the question remains of how this information can be used. In many applications requiring to discriminate among different experimental conditions, e.g. in order to obtain quantitative indices of the effect of a particular stimulus on the time course of the signal, it may be useful to focus on time varying measures obtained by integration on the 

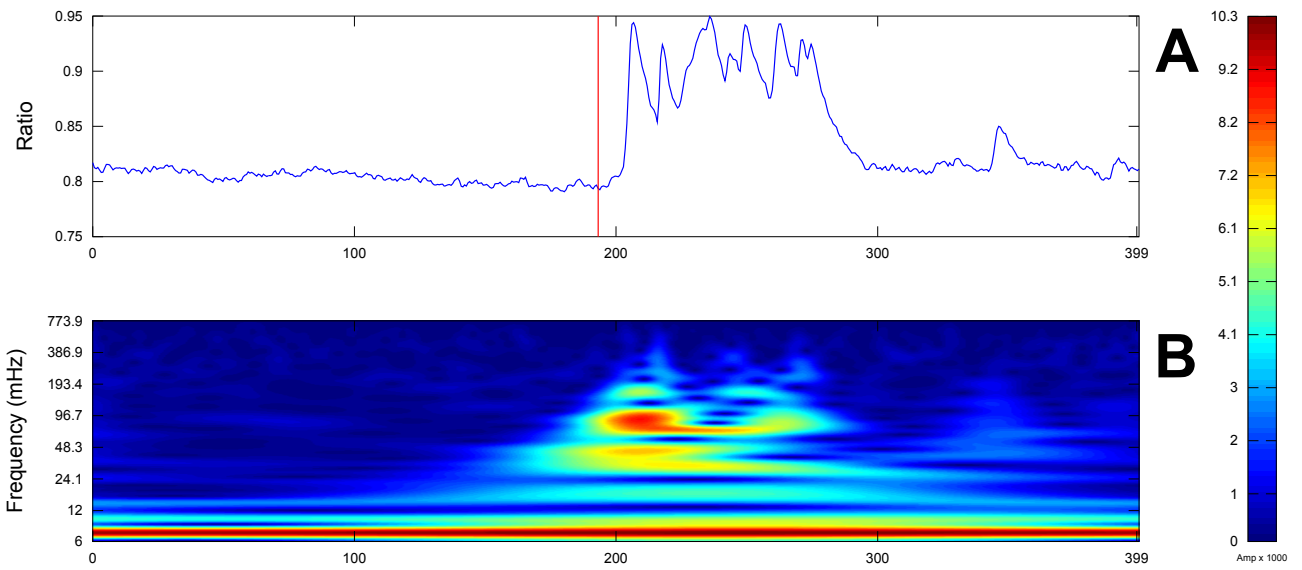

Figure 2: $\mathbf{A}$ : oscillations in $\left[\mathrm{Ca}^{2+}\right]_{c}$ observed in response to stimulation of a chick CG glial cells with $10 \mathrm{nM}$ NAADP. A vertical line marks the starting time of perfusion with the agonist. B: $|W(t, \nu)|$ : amplitude of the wavelet transform as a function of time and frequency.

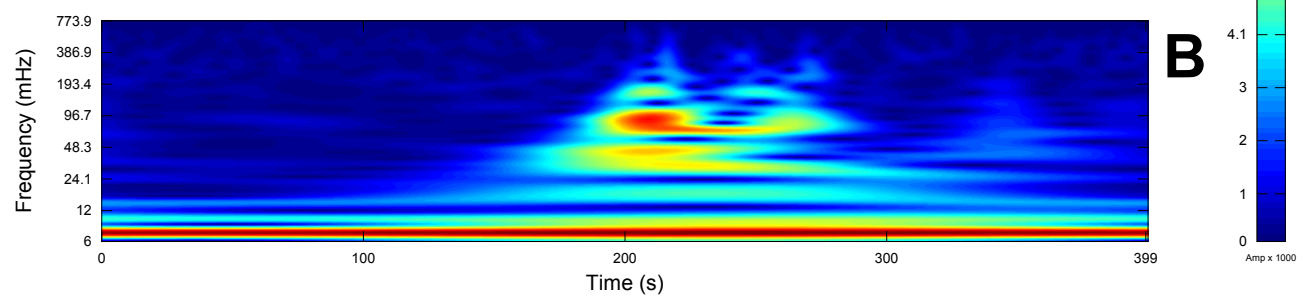

frequency line, or, conversely, measures that depend solely on the frequencies making up the signal.

In Fig. 3 the upper and left insets represent the result of the integration of $|W(t, \nu)|^{2}$ along frequency and time respectively. In other words the top of the figure displays the so called energy density $E(t)$ (Bussow, 2007)

$$
E(t)=\int|W(t, \nu)|^{2} d \nu,
$$

in which all contributions of all frequencies are integrated to provide a function of time. Note that $E(t)$ starts increasing before the application of the stimulus: this is an effect of the spreading of the maxima at low frequencies, remarked before. On the left the power spectrum is represented:

$$
P(\nu)=\int|W(t, \nu)|^{2} d t .
$$

Note that this power spectrum is similar, but not the same, to the one obtained with a Fourier transform; a comparison is shown in Fig. 4.

In the next sections methods will be presented to compute differences between signals obtained in different experimental conditions, by making use of appropriate indices in time and frequency spaces, respectively. 

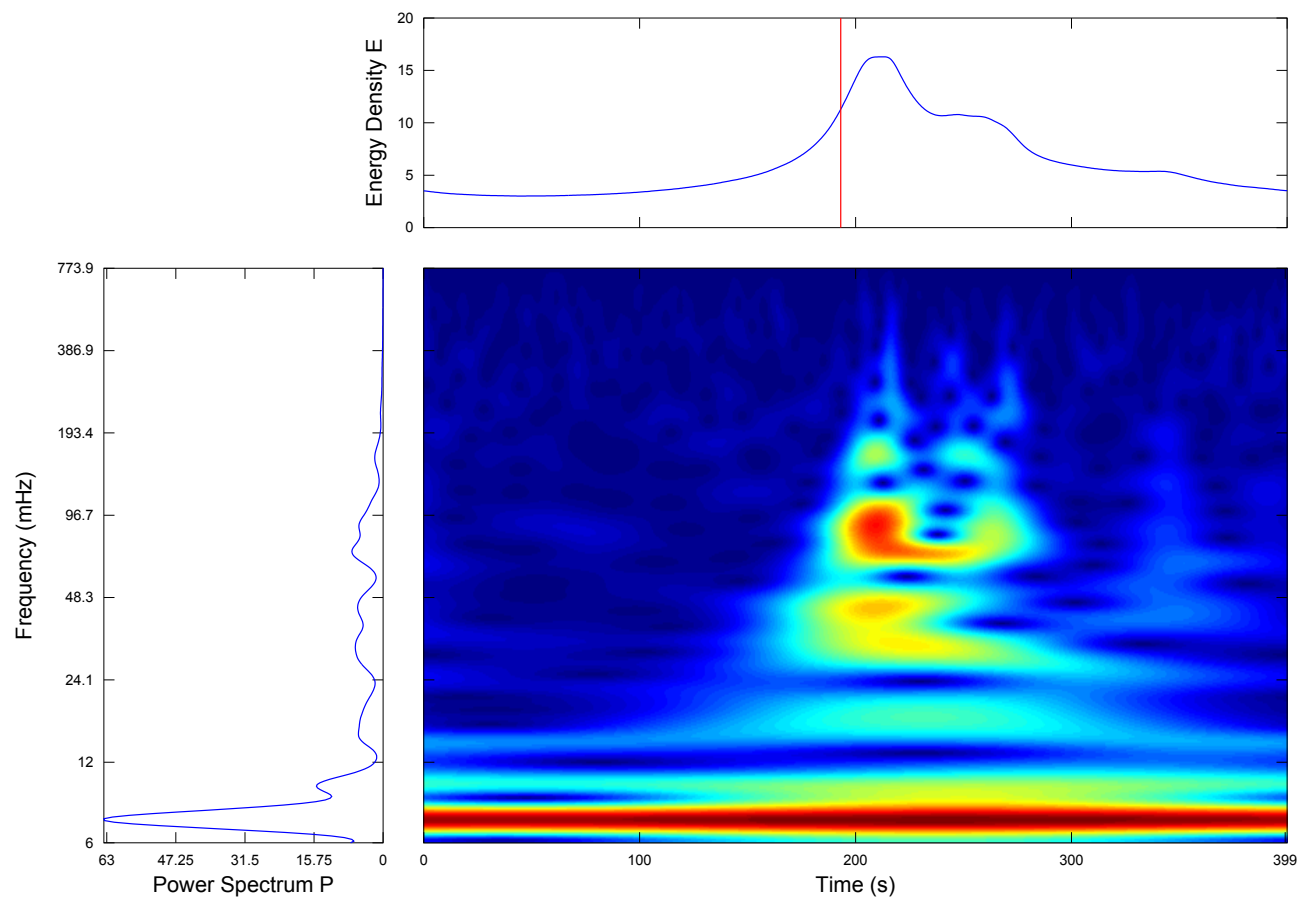

Figure 3: Projections. The central box shows the modulus of the wavelet transform. In the insets: on the left the power spectrum as defined in Eq. (7) and on the top the energy density (see Eq. (6)). 


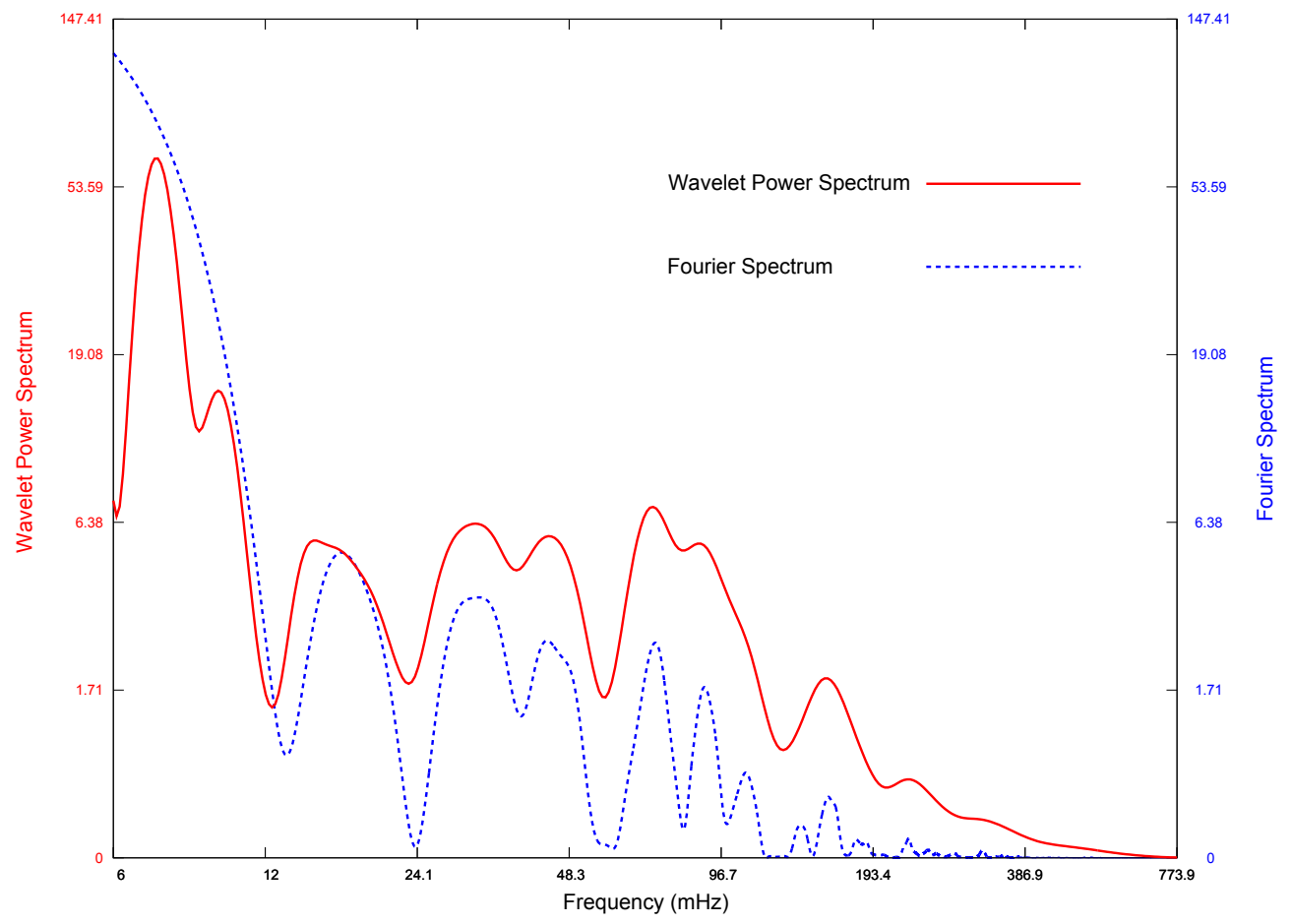

Figure 4: Fourier and wavelet spectra superimposed in a log-log coordinates: the wavelet power spectrum results in a smoother version of the Fourier spectrum, overemphasizing high frequencies. 


\subsection{Focusing on time}

In most cases of interest the main contributions to energy density, at each time point, are concentrated around a few maxima (see Fig. 2), that arise precisely in correspondence with the most relevant events in the signal such as sharp peaks or oscillatory bursts, and these events are characterized by the occurrence of relatively high frequency components.

In order to discriminate between results obtained in different experimental conditions a suitable representation of the signal must take into account both aspects. This can be done, for instance, by summing the contributions of the maxima of $|W(t, \nu)|$ along the $\nu$ axis, weighted by the corresponding values of $\nu$. Maxima of $|W(t, \nu)|$ along the frequency axis can be obtained by the conditions

$$
\left\{\begin{array}{l}
\frac{\partial}{\partial \nu}|W|=0 \\
\frac{\partial^{2}}{\partial \nu^{2}}|W|<0
\end{array},\right.
$$

and a new index $J$ of energy density can ben defined as

$$
J(t)=\frac{1}{2 \epsilon} \int_{t-\epsilon}^{t+\epsilon} \sum_{i=1}^{n(\tau)}\left|W\left(\tau, \nu_{i}\right)\right|^{2} \nu_{i}(\tau) d \tau
$$

where $\left\{\nu_{i}(\tau)\right\}$ is exactly the set of directional local maxima of $W$ along the $\nu$ axis, at time $\tau$. Since the number of these maxima changes in time, the parameter $n$ is expressed as a function of $\tau$. Integration simply serves to regularize the index, by avoiding abrupt variations due to discontinuities of frequency paths. The index $J$, calculated using the signal of Fig. 2, is shown in Fig. 5.

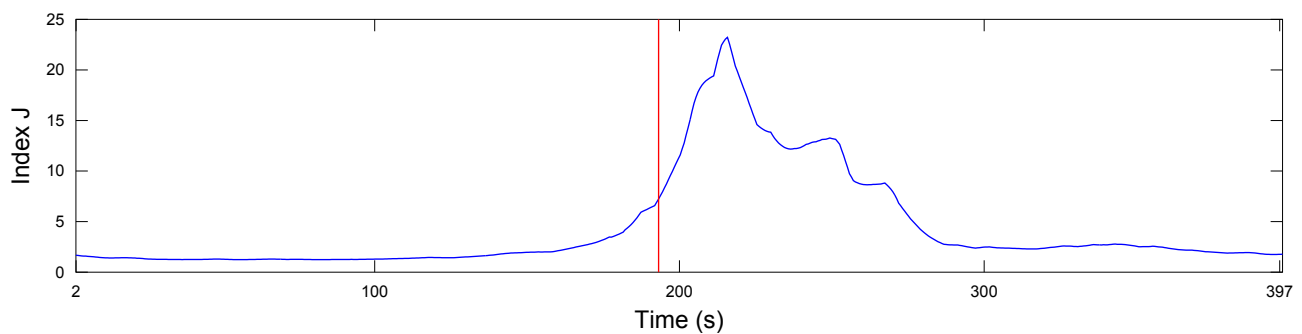

Figure 5: Time course of the index $J$, computed applying Eq. (9) to the modulus of the wavelet transform shown in Fig. 2. 
The index $J$ gives a good quantitative evaluation of the effect of the agonist on the oscillatory activity shown in Fig. 2; the initial peak reflects the greater amplitude of the oscillations in the first part of the burst.

A comparison with $E$ (compare with the inset in Fig. 3) shows that $J$ provides a representation that discriminates better between pre- and poststimulus activity: in particular the maximum is sharper and $J$ starts increasing after the application of the stimulus. This is because the index $J$ has been obtained by taking into account the peaks of the amplitudes weighted by the frequencies, so that higher frequency components have a greater weight. Since high frequencies are better localized in time, this approach provides a reduction of the effect due to the spreading at low frequencies; in other words it enhances the components better resolved in time minimizing the effects of delocalization at low frequencies. The obvious trade-off is that low frequencies are somehow underrepresented; however, this is not a serious flaw since, as stated above, low frequencies correspond just to a global oscillatory trend of the signal.

A global measure can be derived simply by taking the time average of $J$,

$$
\bar{J}=\frac{1}{\delta t} \int_{t_{i}}^{t_{f}} J(t) d t,
$$

where $\delta t=t_{f}-t_{i}$ is the duration of the signal.

Suppose we are given two signals $f_{1}$ and $f_{2}$, then the corresponding indices $\bar{J}_{1}$ and $\bar{J}_{2}$ can be used to derive a measure of the difference in the oscillatory components, for instance by defining

$$
r_{J}=\frac{\bar{J}_{2}}{\bar{J}_{1}} .
$$

Thus $r_{J}$ is a measure of the activity variation of the whole trace: $r_{J}>1$ stands for an enhancement, while $0<r_{J}<1$ corresponds to a decrease (note that since $J(t)$ is a positive defined value, $r_{J}$ too is always positive).

As an example consider again the signal of Fig. 2 and let $\delta t_{1}, \delta t_{2}$ be the intervals before and after the application of the stimulus, respectively. Then $\bar{J}_{1}, \bar{J}_{2}$ are the temporal averages of $J$ before and after the stimulus, and their values are $\bar{J}_{1}=1.86, \bar{J}_{2}=6.74$. The ratio $r_{J}=3.63$ is relatively large, showing that $\bar{J}_{1}, \bar{J}_{2}$ are able to provide a measure of the effect of the stimulus on the signal time course; by comparison note that the ratio of preand post-stimulus averages of the density of energy $E(t)$ is just 1.89 . 
In conclusion, by means of the index $J(t)$, we can obtain an instantaneous estimate of the oscillatory activity of the signal and by computing its temporal mean $\bar{J}$, we can assign a single scalar value to each arbitrarily defined temporal interval (for instance a pre-treatment value $\bar{J}_{1}$ and a post-treatment value $\left.\bar{J}_{2}\right)$. Finally, the ratio $r_{J}$ between $\bar{J}_{2}$ and $\bar{J}_{1}$ is a global measure of the variation of the oscillatory trend within a single trace; since it is defined as a ratio between two temporal mean quantities, any basal component can be ignored.

\subsection{Focusing on frequencies}

Consider the time average, in an interval $\delta t$, of the modulus $|W(t, \nu)|$ of the wavelet transform:

$$
\mathbf{V}(\nu)=\frac{1}{\delta t} \int_{t_{i}}^{t_{f}}|W(t, \nu)| d t
$$

This average is a function of $\nu$ that can be considered to be representative of the frequency spectrum within the interval. Note also that $\mathbf{V}$ can be regarded as an infinite-dimensional vector, whose components are the frequencies $\nu$, and component values are given by $\mathbf{V}(\nu)$; therefore some tools of vector analysis can be applied here. For instance it is possible to determine a distance $d$, in the frequency space, between signals recorded in different experimental conditions.

Consider two signals $f_{1}, f_{2}$ of duration $\delta t_{1}, \delta t_{2}$ respectively, and the corresponding vectors $\mathbf{V}_{1}(\nu)$ and $\mathbf{V}_{2}(\nu)$. The distance $d$ is defined as

$$
d=\left[\int_{0}^{+\infty}\left[\mathbf{V}_{2}(\nu)-\mathbf{V}_{1}(\nu)\right]^{2} d \nu\right]^{1 / 2},
$$

and it is straightforward to show that

$$
d=\left[\left\|\mathbf{V}_{1}\right\|^{2}+\left\|\mathbf{V}_{2}\right\|^{2}-2\left\|\mathbf{V}_{1}\right\|\left\|\mathbf{V}_{2}\right\| \cos \theta\right]^{1 / 2},
$$

where

$$
\|\mathbf{V}\|=\left[\int_{0}^{+\infty}[\mathbf{V}(\nu)]^{2} d \nu\right]^{1 / 2},
$$

is the norm of $\mathbf{V}(\nu)$, and

$$
\cos \theta=\frac{1}{\left\|\mathbf{V}_{1}\right\|\left\|\mathbf{V}_{2}\right\|} \int_{0}^{+\infty} \mathbf{V}_{1}(\nu) \cdot \mathbf{V}_{2}(\nu) d \nu
$$


Equation (14) can be rewritten as

$$
d=\left[\Delta^{2}+2\left\|\mathbf{V}_{1}\right\|\left\|\mathbf{V}_{2}\right\|(1-\cos \theta)\right]^{1 / 2},
$$

where $\Delta$ is the modulus of the difference between the norms, namely $\Delta=$ ||$\left|\mathbf{V}_{1}\|-\| \mathbf{V}_{2} \|\right|$.

In practice integrals (13), (15) and (16) must be replaced by summations over a discrete and finite set of frequencies, ranging from $\nu_{\text {low }}=\frac{1}{T}$ to Nyquist frequency $\nu_{n y q}=\frac{1}{2 \delta \tau}$, where $T$ is the total recording time, while $\delta \tau$ is the sampling time.

In reference to equation (17), the distance $d$ depends on two factors: the difference $\Delta$ between the norms of vectors $\mathbf{V}_{1}$ and $\mathbf{V}_{2}$ and their relative orientations. Consider the case $\theta=0$ : the vectors are parallel (in the infinitedimensional space), that is to say that they have the same frequency content, the only difference being a scale factor. In this case $d=\Delta$. Conversely if $\theta=\pi / 2$ the scalar product is zero, that is $\mathbf{V}_{1}, \mathbf{V}_{2}$ are orthogonal and that means that one signal is made up of frequencies that have zero amplitude in the other one. Now $d=\left[\left\|\mathbf{V}_{1}\right\|^{2}+\left\|\mathbf{V}_{2}\right\|^{2}\right]^{1 / 2}$.

However, some care must be taken when considering the distance $d$. For instance, if $\left\|\mathbf{V}_{1}\right\|$ and $\left\|\mathbf{V}_{2}\right\|$ are equal $(\Delta=0)$ and very large even a small angular difference $\theta$ can lead to a large distance $d$. Thus, in this framework, it is appropriate to consider together with $d$ and $\Delta$ the angular difference $\theta$, that does not depend on the norms.

An example is shown in Fig. 6. As before $\delta t_{1}, \delta t_{2}$ are the pre- and poststimulus time intervals and $\mathbf{V}_{1}$ and $\mathbf{V}_{2}$ are the corresponding vectors. It is apparent from Fig. 6 that the difference between vectors $\mathbf{V}_{1}$ and $\mathbf{V}_{2}$ is due to both factors: $\mathbf{V}_{2}$ is larger than $\mathbf{V}_{1}$ at all frequencies, and at intermediate frequencies it has peaks that do not appear in $\mathbf{V}_{1}$ : here $d=3.43, \Delta=2.11$, that is $\Delta$ contributes to the $60 \%$ of the distance $d$.

This approach can unravel subtle differences in the spectral components of the signal in different experimental conditions, that may reflect different mechanisms of generation of calcium oscillations.

\subsection{Computational considerations}

In order to carry out the analysis a software, called KYM, has been developed under the freely redistributable GNU Octave environment (Eaton et al., 2008). Octave is a high-level language, primarily intended for numerical computations. It provides a convenient command line interface for solving linear 


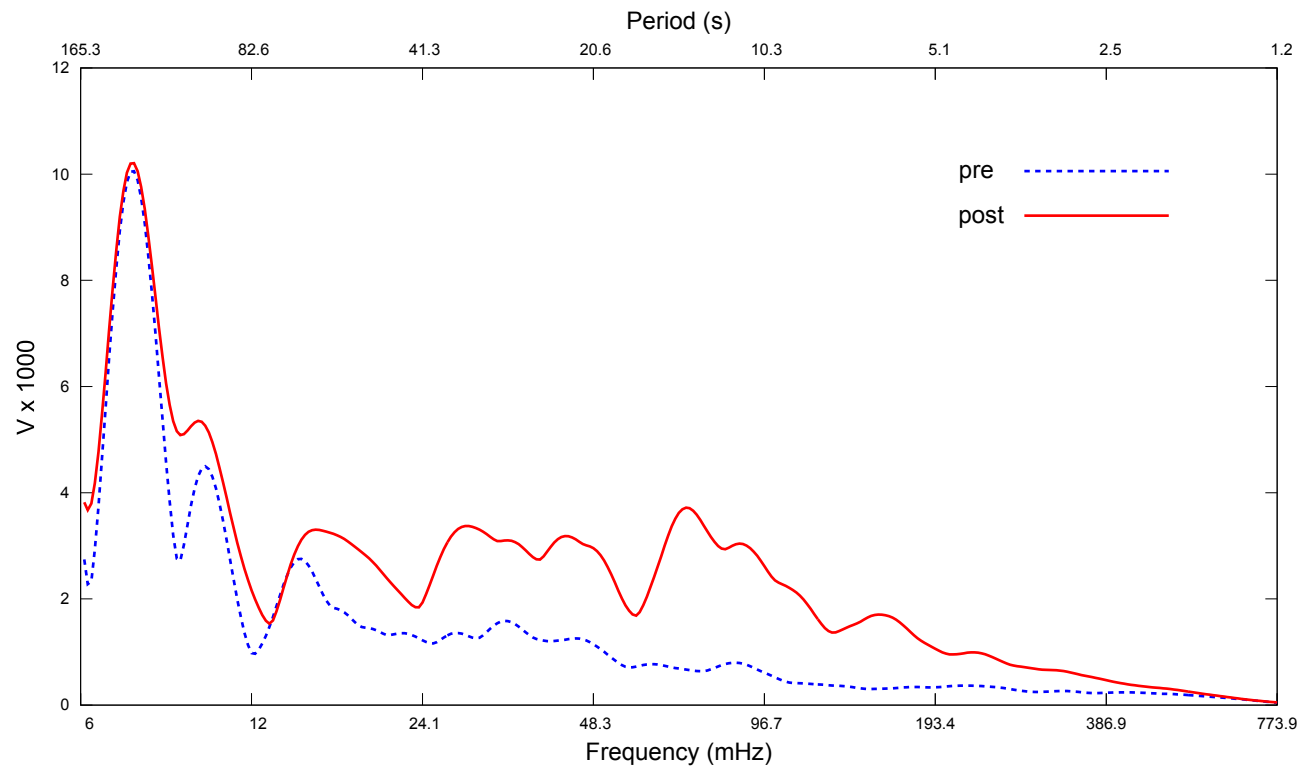

Figure 6: Vector analysis. The dashed line represents the vector $\mathbf{V}_{1}$, computed from the pre-stimulus signal, whereas the continuous line refers to $\mathbf{V}_{2}$, corresponding to the post-stimulus signal. 
and nonlinear problems numerically, and for performing other numerical experiments using a language that is mostly compatible with other popular high-level language environments. As it is easily expandable and customizable we wrote KYM as user-defined functions in Octave's 3.2.3 own language and tested it on both Microsoft and Linux Debian systems.

At the present KYM is made up of $14 . \mathrm{m}$ files each containing a single function, for a total of about 1700 code lines, but only 4 of them (VX, WT, PD, FEAT) need to be directly managed by the end user. The other ones are auxiliary functions invoked on the fly by the mains. All numerical and graphic results presented in this work have been obtained by means of KYM routines but only a small part of the features is shown in the present work. The architecture allows component reuse and quick prototyping of new tracks processing algorithms, making new developments and further optimizations easier to be implemented. KYM has a command-line user-interaction that has not been developed taking into account the end users and, therefore, a future effort will be to make a user-friendly interface.

In order to use KYM, data need to be stored in a .csv (comma separated values) file. It must contain the vector of time samples as first column, while the time courses of the fluorescence intensity for each cell (or region of interest) must fill the next columns in the matrix. Some parameters can be passed as argument to the main functions, in order to specify time units, points at which changes in the extracellular medium have been performed, threshold levels, and so on. Actual wavelet computation consists in the usual method that implements a time convolution as a product in the Fourier transformed domain; code for this algorithm can be downloaded at http: //www-stat.stanford.edu/ wavelab/. Peak detection uses a technique that is based on images dilation (see for instance http://www.mathworks. com/matlabcentral/fileexchange/authors/26510/). The rest of code has been written and developed ad hoc to perform the analysis presented here. At the moment KYM does not implement any algorithm to detect the cone of influence; this problem will be addressed in an upgraded version of the software.

We make KYM available as supplementary material: as it is an open source code one can inspect it to see exactly what algorithms have been used, and then modify the source to produce a better code or to satisfy other particular needs. Users may redistribute it and/or modify it under the terms of the GNU General Public License (GPL) as published by the Free Software Foundation. Because KYM is a free software users are encouraged to help 
make Octave more useful by writing and contributing additional functions for it, and by reporting any problems they may have.

The most up-to-date version can be downloaded from the well-established public-domain repository SourceForge (http://sourceforge.net/projects / $\mathrm{kym} /$ ). The .m files come with an extended documentation in the heading, explaining the syntax and the meaning of each function and related arguments.

To our knowledge this is the first open source tool specifically dedicated to the analysis of the time course of cellular calcium signals and more generally of oscillatory signals recorded by means of fluorescent dyes from biological systems.

\subsection{Experimental procedures}

Chick ciliary ganglion cells were obtained from 7 day embryos and maintained for 1-3 days in a chemically defined N2 medium as previously described (Distasi et al., 1998). Cells were loaded for $30 \mathrm{~min}$ at $37^{\circ} \mathrm{C}$ with $0.5 \mu \mathrm{M}$ Fura-2 (Invitrogen, USA), transferred in a perfusion chamber (Bioptechs, USA) and mounted on an inverted microscope (Eclipse TE 300, Nikon, Japan). Experiments were performed at a temperature of $37^{\circ} \mathrm{C}$. During experiments cells were continuously superfused by means of a gravity microperfusion system combined with electrovalves to allow switching between different solutions. The control solution was a standard Tyrode solution of the following composition, in $\mathrm{mM}$ : $\mathrm{NaCl}, 154 ; \mathrm{KCl}, 4 ; \mathrm{CaCl}_{2}, 42 ; \mathrm{MgCl}_{2}, 1$; $\mathrm{N}$-(2-Hydroxyethyl)-piperazine-N'-ethanesulfonic acid (HEPES), 54; glucose, 5.5; $\mathrm{NaOH}$ to $\mathrm{pH}$ 7.4. $\left[\mathrm{Ca}^{2+}\right]_{c}$ measurements were performed exciting the dye Fura-2 alternatively at $340 \mathrm{~nm}$ and $380 \mathrm{~nm}$ for $100 \mathrm{~ms}$ by means of a monochromator (Polychrome IV, T.I.L.L. Photonics GmbH, Germany), and recording emission at $510 \mathrm{~nm}$. Images were acquired with a cooled CCD camera (SensiCam, PCO, Germany) and stored on a computer. Fluorescence was determined from regions of interest (ROI) covering single glial cell bodies. The use of a ratiometric probe allowed to rule out any effect on signal amplitudes of dye loading and potential changes in fluorescence emission during the experiments. Dye excitation, image acquisitions and ROI analysis protocols were performed with Axon Imaging Workbench software (Axon Instruments, USA). Satellite glial cells were identified on morphological and functional criteria as previously reported (Bernascone et al., 2010) and NAADP was synthesized and purified as described in Billington and Genazzani (2000). 


\section{Results}

The approach described above was tested on a group of traces obtained from the same experiment of the trace shown in Fig. 2 (i.e. ciliary ganglion glial cells challenged with $10 \mathrm{nM}$ NAADP). We selected traces showing different patterns in time and for which in some cases the interpretation of the results was not straightforward.

The upper box of Fig. 7 refers to a case in which the occurrence of a change in the oscillatory pattern after the stimulus can be deduced from a visual observation. The index $J$ (middle box) starts to increase with the application of the agonist, the corresponding ratio $r_{J}$ has a relatively high value, $r_{J}=2.43$. Vector analysis (lower box) shows an increase of all frequency components between 10 and $96 \mathrm{mHz}$, and indeed the distance is mainly due to differences between the norms $\Delta$ since amplitude enhancement affects all frequency components: $d=2.86$ and $\Delta=2.33$.

On the contrary the trace displayed in the upper box of Fig. 8 shows oscillatory activity both before and after the stimulus with a random component, and it is therefore difficult to extract information, by means of direct observation, on the occurrence of a response. However, the relatively large values taken by the index $J$, with $r_{J}=1.64$, points to an enhancement of the oscillatory pattern; vector analysis reveals that the growth of $J$ is due to a different modulation in the post-stimulus interval and in particular to an increase of the components in the frequency range $24-98 \mathrm{mHz}$. The fact that most of the distance $d$ between $\mathbf{V}_{2}$ and $\mathbf{V}_{1}$ is due to the variation of spectral contents in the pre- and post-stimulus intervals is shown by the small contribution of $\Delta=0.51$ to $d=1.61$.

In the trace of Fig. 9 after the administration of the agonist a clear response can be observed, consisting of a sharp transient of $\left[\mathrm{Ca}^{2+}\right]_{c}$ followed by a plateau with limited oscillations. The wavelet transform of this type of signal is mainly formed by the contribution of a low frequency component that, as explained earlier, is poorly discriminated in time; thus is not surprising that the index $J$ starts to slowly increase before the stimulation, and it is characterized by a lower value of ratio $r_{J}\left(r_{J}=1.28\right)$. Note that the step size increase in the signal produces large values of $\mathbf{V}_{1}$ and $\mathbf{V}_{2}$ that, in turn, give rise to a relatively large distance $(d=2.97)$ even in absence of a significant difference between the spectral content of vectors $\mathbf{V}_{1}$ and $\mathbf{V}_{2}$, as explained before. Indeed both the norm and angular differences are small: $\Delta=0.79, \theta=0.24 \mathrm{rad}$. 

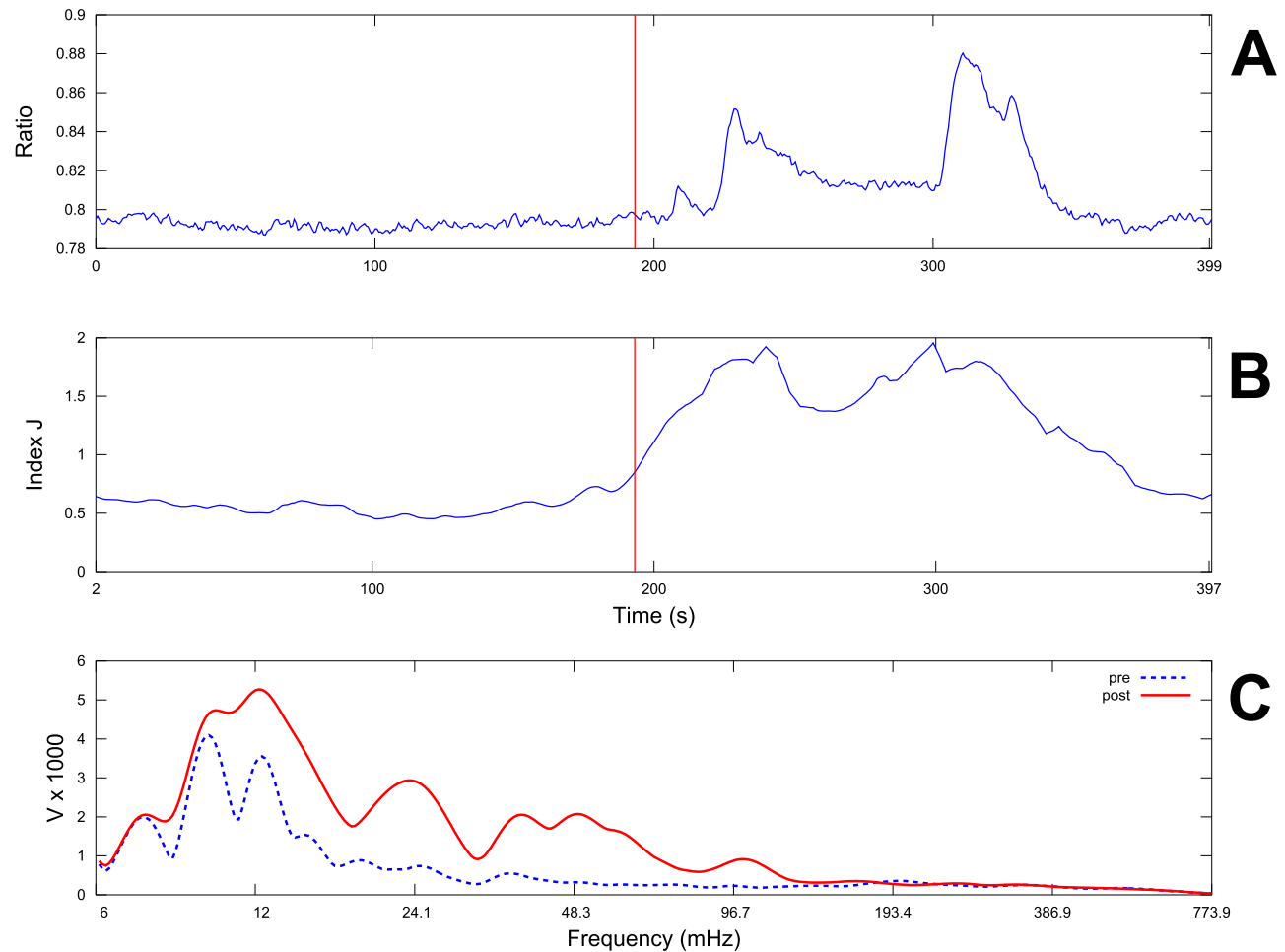

Figure 7: Second trace. A: oscillations in $\left[\mathrm{Ca}^{2+}\right]_{c}$ observed in response to stimulation of a CG glial cells with $10 \mathrm{nM}$ NAADP. B: time course of the index $J$. C: the dashed line represents the vector $\mathbf{V}_{1}$, computed from the pre-stimulus signal, whereas the continuous line refers to $\mathbf{V}_{2}$, corresponding to the post-stimulus signal. 

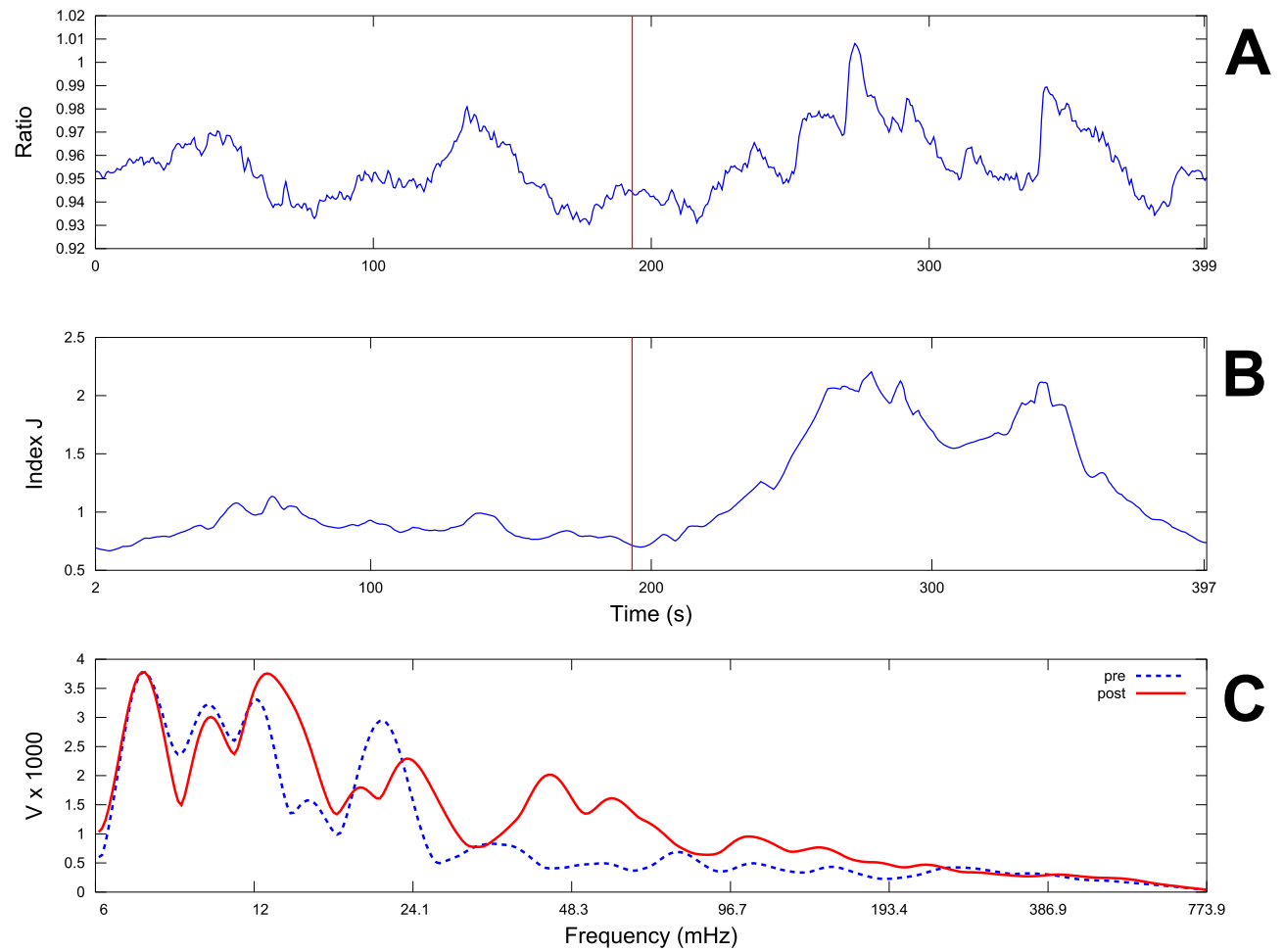

Figure 8: Third trace. A: oscillations in $\left[\mathrm{Ca}^{2+}\right]_{c}$ observed in response to stimulation of a CG glial cells with $10 \mathrm{nM}$ NAADP. B: time course of the index $J$. C: the dashed line represents the vector $\mathbf{V}_{1}$, computed from the pre-stimulus signal, whereas the continuous line refers to $\mathbf{V}_{2}$, corresponding to the post-stimulus signal. 

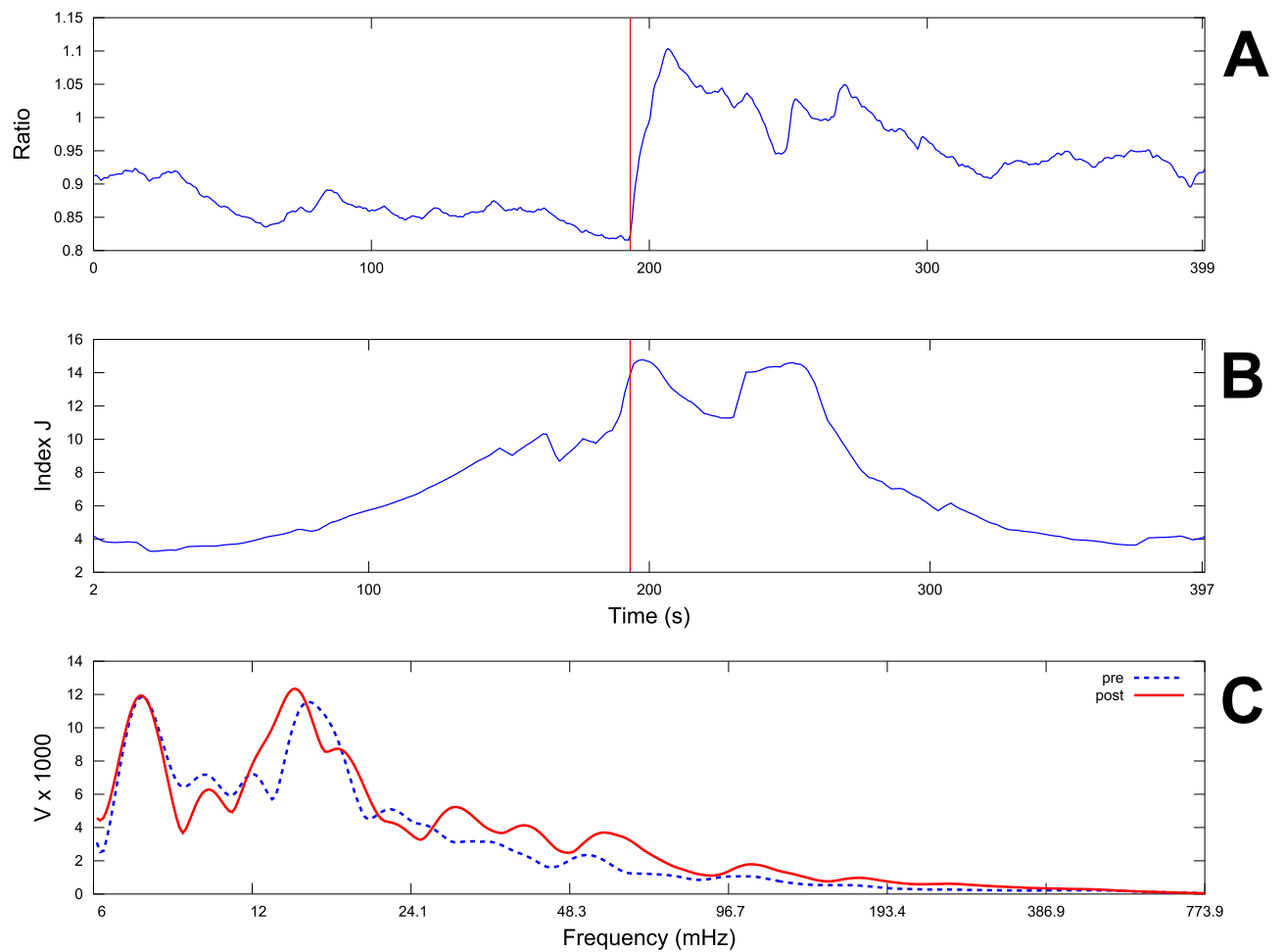

Figure 9: Fourth trace. A: oscillations in $\left[\mathrm{Ca}^{2+}\right]_{c}$ observed in response to stimulation of a CG glial cells with $10 \mathrm{nM}$ NAADP. B: time course of the index $J$. C: the dashed line represents the vector $\mathbf{V}_{1}$, computed from the pre-stimulus signal, whereas the continuous line refers to $\mathbf{V}_{2}$, corresponding to the post-stimulus signal. 
The observation that changes in the frequency content span the same range in all four cells shown (Figs. 6, 7, 8, 9) can be formalized in a more objective quantification of the effect of the agonist, simply defining a new function:

$$
R(\nu)=\frac{1}{N} \sum_{i=1}^{N}\left(\frac{V_{2}(\nu)}{V_{1}(\nu)}\right)_{i},
$$

where the sum runs over cells number: thus $R$ is the mean ratio of post- to pre-treatment spectra.

The function $R$ represents the average spectral distribution of the oscillatory activity enhancement, following agonist administration. The function we obtained is clearly a non-flat distribution (Fig. 10). In particular it

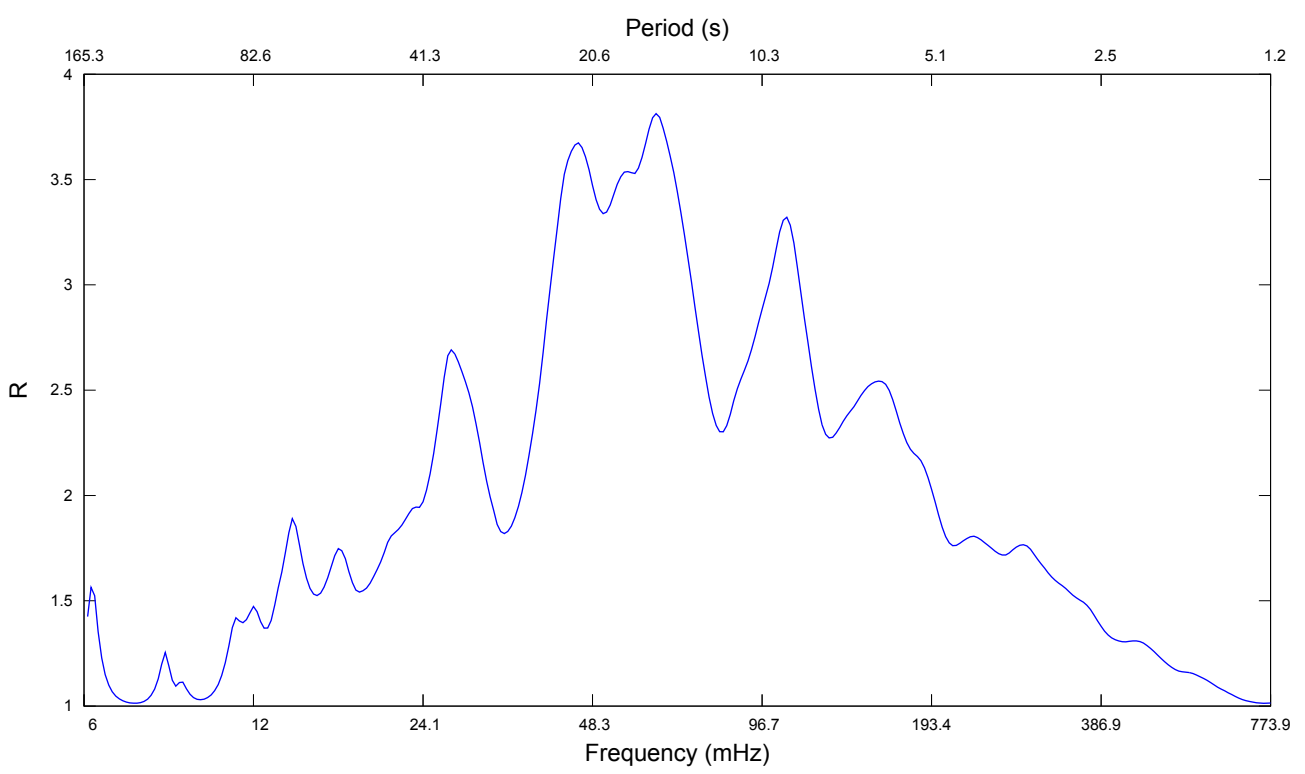

Figure 10: The function $R$ as given by Eq. (18); here $N=4$.

presents its highest values inside that same range of frequencies previously mentioned $(24-193 \mathrm{mHz})$, with a peak centered on $50 \mathrm{mHz}$. Even if the number of cells used in this data analysis is small $(N=4)$, function (18) turns out to be able to discriminate the frequencies involved in cellular oscillatory response, but is reasonable to think that this function would have been more smooth and more peaked if we had kept into account a larger number of traces. A step in this direction is presented in the following section. 


\subsection{Statistical validation}

It has been observed in section 3.1 that the ratio $r_{J}$ provides a global measure of the effect of the stimulus on the signal time course and that, in particular, $r_{J}>1$ should correspond to an enhancement of $\left[\mathrm{Ca}^{2+}\right]_{c}$ oscillations and $0<r_{J}<1$ to an inhibition.

To provide a statistical validation of $r_{J}$ values, we have considered a set of 36 traces from 6 different experiments similar to those described above, the only difference being that now CG glial cells were bathed in a Tyrode standard solution and then stimulated with a higher dose of NAADP $(1 \mu \mathrm{M}$; data not shown). For each trace the corresponding $r_{J}$ was computed, thus generating a sample $\left\{r_{J}\right\}$ whose histogram is shown in Fig. 11.A.

While it is clear that $r_{J}$ is not normally distributed, as it could be expected since its range is from 0 to $+\infty$, a distribution more closely resembling a normal can be obtained by considering a transformation from $r_{J}$ to $\log r_{J}$ (see Fig. 11.B). Indeed a Q-Q plot (Fig. 11.C) shows the data lying very close to the bisector; a Shapiro-Wilk (SW) normality test gives $p=0.639$, much higher than the threshold for the rejection of normality hypothesis $\alpha=0.05$.

It follows that we can assume the distribution of $r_{J}$ to be log-normal, and this holds also for the sampling distribution obtained by application to our sample of a bootstrap procedure with $10^{6}$ iterations.

We have then computed the mean and corresponding confidence interval $(C I)$ for the original data and the bootstrap distribution, and both approaches lead to the same numerical results. In both cases $\bar{r}_{J}=2.49$, and $C I=[2.02 ; 3.04]$ is the $99 \%$ confidence interval; namely there is a probability $p \geq 0.99$ that the true value of the index $r_{J}$ is comprised between 2.02 and 3.04 .

If $r_{J}$ did not measure the effect of the stimulus on the oscillatory activity of $\left[\mathrm{Ca}^{2+}\right]_{c}$ time course any departure from $r_{J}=1$ would be ascribed solely to chance; since $r_{J}=1$ is outside the $99 \%$ confidence interval we can reject the null hypothesis that $r_{J}=1$ is the true value of index $r_{J}$.

With this approach, therefore, we provide a statistical validation of a significant difference between pre- and post-stimulus condition in a whole set of data, that is to say that the results reflect the occurrence of a response of the cell population to the stimulus.

For comparison we have computed the index $J$ using $W$ values of all frequencies, and not just those corresponding to the maxima; the index computed this way does not give better results, thus confirming our hypothesis 

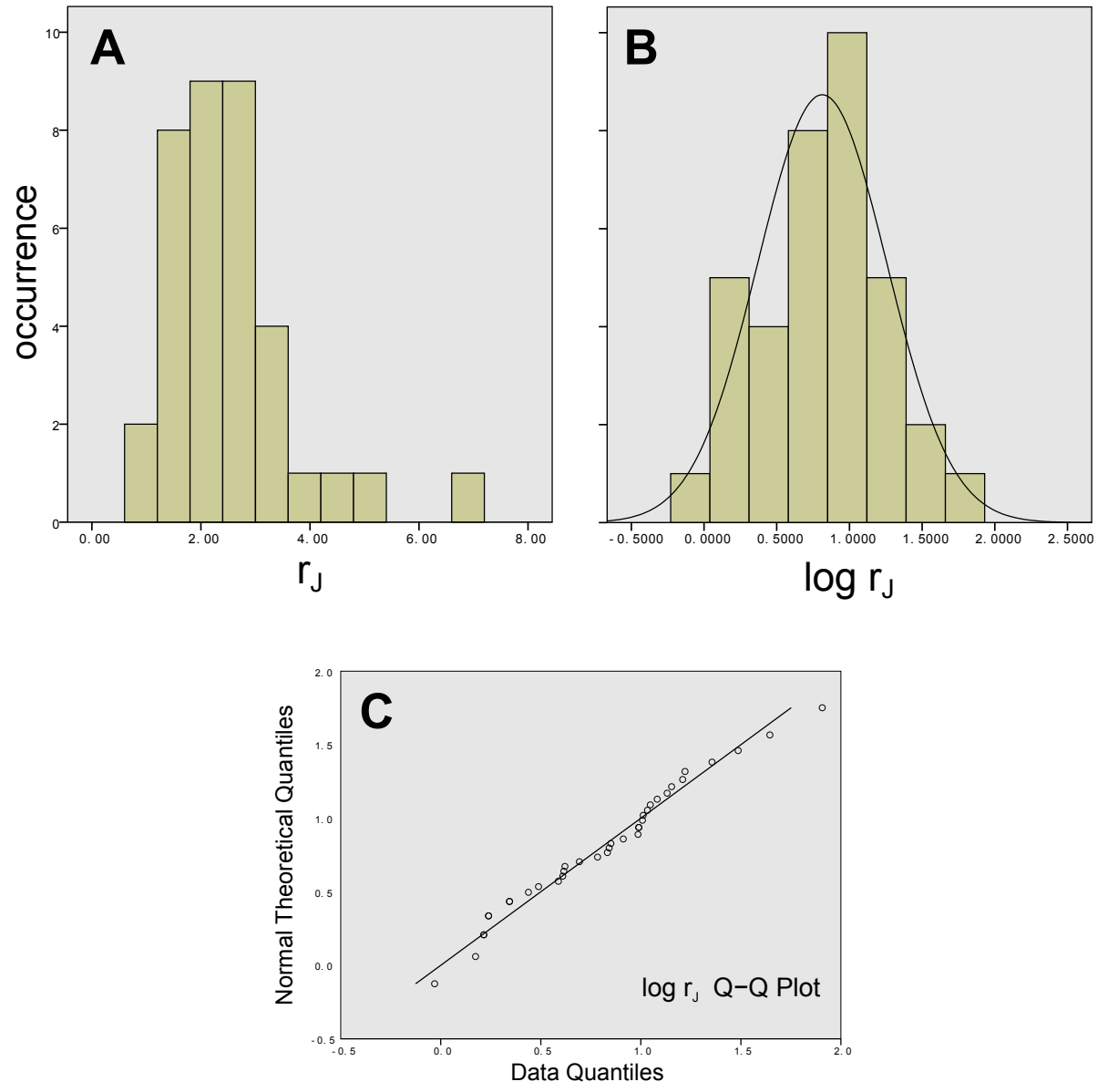

Figure 11: Statistical analysis. A: Histogram of $r_{J}$ values computed from 36 different traces. The average of $r_{J}$ is $\bar{r}_{J}=2.49$. B: Histogram of the new variable $\log r_{J}$. C: Q-Q plot of the variable $\log r_{J}$. 
that maxima contain most of the relevant information about the frequency composition.

\section{Conclusion}

Wavelet analysis is a well known tool to study the properties of a signal and to extract information about the temporal changes of its oscillatory structure. However there are applications, such as analysis of changes in the cytosolic free calcium concentration $\left[\mathrm{Ca}^{2+}\right]_{c}$, that require to discriminate between different patterns of signal activity either in space or in time. Here an approach has been presented in which by separating time and frequency domains, indices have been derived to characterize quantitatively changes in the oscillatory behavior of $\left[\mathrm{Ca}^{2+}\right]_{c}$ with respect to different experimental conditions, in particular pre- and post-stimulus conditions. This approach has been tested on a set of experimental recordings showing heterogeneous patterns of activation, and it has been proved to be able to discern subtle differences between them.

In particular the procedure described here represents a general method that allows a rigorous and automated characterization of both cellular spontaneous activity and effects of agonists in terms of oscillatory behaviors. This approach has been used here for discriminating between different patterns in time, but it could be highly useful in different contexts, such as in analyzing spatial differences in signals recorded from subdomains of the same cell.

\section{Acknowledgments}

We thank Prof. A. Genazzani for the gift of NAADP used in the experiments.

\section{References}

Antonini M, Barlaud M, Mathieu P, Daubechies I. Image coding using wavelet transforms. IEEE Trans Image Process 1992;1:205-20.

Bernascone S, Erriquez J, Ferraro M, Genazzani AA, Distasi C. Novel adenosine and cAMP signalling pathways in migrating glial cells. Cell Calcium 2010;48:83-90. 
Billington RA, Bellomo EA, Floriddia EM, Erriquez J, Distasi C, Genazzani AA. A transport mechanism for NAADP in a rat basophilic cell line. FASEB J 2006;20:521-3.

Billington RA, Genazzani AA. Characterization of NAADP $(+)$ binding in sea urchin eggs. Biochem Biophys Res Commun 2000;276:112-6.

Bussow R. An algoritm for the continuous Morlet wavelet transform. Mech Syst Signal Process 2007;21:2970-9.

Constantin S, Caligioni CS, Stojilkovic S, Wray S. Kisspeptin-10 facilitates a plasma membrane-driven calcium oscillator in gonadotropin-releasing hormone-1 neurons. Endocrinology 2009;150:1400-12.

Daubechies I. Ten lectures on wavelets. Philadelphia, Penn: Society for Industrial and Applied Mathematics, 1992.

Distasi C, Torre M, Antoniotti S, Munaron L, Lovisolo D. Neuronal survival and calcium influx induced by basic fibroblast growth factor in chick ciliary ganglion neurons. Eur J Neurosci 1998;10:2276-86.

Eaton JW, Bateman D, Hauberg S. GNU Octave manual. UK: Network Theory Ltd, 2008.

Frey N, McKinsey TA, Olson EN. Decoding calcium signals involved in cardiac growth and function. Nat Med 2000;6:1221-7.

Genazzani AA, Billington RA. NAADP: an atypical Ca2+-release messenger? Trends Pharmacol Sci 2002;23:165-7.

Goldberg JH, Yuste R. Space matters: local and global dendritic Ca2+ compartmentalization in cortical interneurons. Trends Neurosci 2005;28:15867.

Gorbunova YV, Spitzer NC. Dynamic interactions of cyclic AMP transients and spontaneous $\mathrm{Ca}(2+)$ spikes. Nature 2002;418:93-6.

Goupillaud P, Grossman A, Morlet J. Cycle-Octave and related transforms in seismic signal analysis. Geoexploration 1984;23:85-102.

Lokenath D. Brief historical introduction to wavelet transforms. Int J Math Educ Sci Technol 1998;29:677-88. 
Mallat S. A wavelet tour of signal processing. San Diego, Ca: Academic Press, 1999.

Oike M, Droogmans G, Nilius B. Amplitude modulation of $\mathrm{Ca} 2+$ signals induced by histamine in human endothelial cells. Biochim Biophys Acta 1994;1222:287-91.

Prank K, Gabbiani F, Brabant G. Coding efficiency and information rates in transmembrane signaling. Biosystems 2000;1-3:15-22.

Raymond CR, Redman SJ. Spatial segregation of neuronal calcium signals encodes different forms of LTP in rat hippocampus. J Physiol 2006;570:97111.

Suzuki N, Takahata M, Sato K. Oscillatory current responses of olfactory receptor neurons to odorants and computer simulation based on a cyclic AMP transduction model. Chem Senses 2002;27:789-801.

Torrence C, Compo GP. A practical guide to wavelet analysis. Bull Am Meteorol Soc 1998;79:61-78.

Uhlén P. Spectral analysis of calcium oscillations. Sci STKE 2004;258:1-12.

Uhlén P, Fritz N. Biochemistry of calcium oscillations. Biochem Biophys Res Commun 2010;396:28-32.

Wegner F, Both M, Fink RH. Automated detection of elementary calcium release events using the a trous wavelet transform. Biophys J 2006;90:215163.

Wen Z, Zheng JQ. Directional guidance of nerve growth cones. Curr Opin Neurobiol 2006;16:52-8. 


\section{List of Figures}

1 Real and imaginary parts of the Morlet mother wavelet, as given by Eq. (4). . . . . . . . . . . . . . . . . . . . 5

2 A: oscillations in $\left[\mathrm{Ca}^{2+}\right]_{c}$ observed in response to stimulation of a chick CG glial cells with $10 \mathrm{nM}$ NAADP. A vertical line marks the starting time of perfusion with the agonist. B: $|W(t, \nu)|$ : amplitude of the wavelet transform as a function of time and frequency. . . . . . . . . . . . 7

3 Projections. The central box shows the modulus of the wavelet transform. In the insets: on the left the power spectrum as defined in Eq. (7) and on the top the energy density (see Eq.

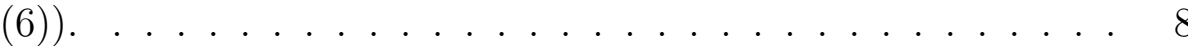

4 Fourier and wavelet spectra superimposed in a log-log coordinates: the wavelet power spectrum results in a smoother version of the Fourier spectrum, overemphasizing high frequencies. 9

5 Time course of the index $J$, computed applying Eq. (9) to the modulus of the wavelet transform shown in Fig. 2. . . . . . . . 10

6 Vector analysis. The dashed line represents the vector $\mathbf{V}_{1}$, computed from the pre-stimulus signal, whereas the continuous line refers to $\mathbf{V}_{2}$, corresponding to the post-stimulus signal. 14

7 Second trace. $\mathbf{A}$ : oscillations in $\left[\mathrm{Ca}^{2+}\right]_{c}$ observed in response to stimulation of a CG glial cells with 10 nM NAADP. B: time course of the index $J$. C: the dashed line represents the vector $\mathbf{V}_{1}$, computed from the pre-stimulus signal, whereas the continuous line refers to $\mathbf{V}_{2}$, corresponding to the poststimulus signal. . . . . . . . . . . . . . . . 18

8 Third trace. A: oscillations in $\left[\mathrm{Ca}^{2+}\right]_{c}$ observed in response to stimulation of a CG glial cells with $10 \mathrm{nM}$ NAADP. B: time course of the index $J$. C: the dashed line represents the vector $\mathbf{V}_{1}$, computed from the pre-stimulus signal, whereas the continuous line refers to $\mathbf{V}_{2}$, corresponding to the poststimulus signal. . . . . . . . . . . . . . 
9 Fourth trace. A: oscillations in $\left[\mathrm{Ca}^{2+}\right]_{c}$ observed in response to stimulation of a CG glial cells with $10 \mathrm{nM}$ NAADP. B: time course of the index $J$. C: the dashed line represents the vector $\mathbf{V}_{1}$, computed from the pre-stimulus signal, whereas the continuous line refers to $\mathbf{V}_{2}$, corresponding to the poststimulus signal. . . . . . . . . . . . . . . . 2 20

10 The function $R$ as given by Eq. (18); here $N=4$. . . . . . 21

11 Statistical analysis. A: Histogram of $r_{J}$ values computed from 36 different traces. The average of $r_{J}$ is $\bar{r}_{J}=2.49$. B: Histogram of the new variable $\log r_{J}$. C: Q-Q plot of the variable

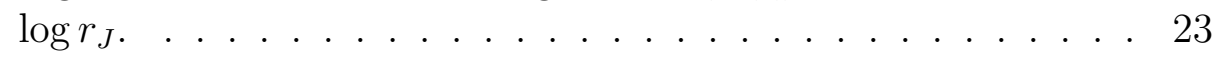

